\title{
Diagnosis and management of neurotrophic keratitis
}

This article was published in the following Dove Press journal:

Clinical Ophthalmology

19 March 2014

Number of times this article has been viewed

\author{
Marta Sacchetti ${ }^{1}$ \\ Alessandro Lambiase ${ }^{2}$ \\ 'Cornea and Ocular Surface Unit, \\ Ospedale San Raffaele di Milano- \\ IRCCS, Milan, ${ }^{2}$ Ophthalmology, \\ University La Sapienza of Rome, Italy
}

Correspondence: Alessandro Lambiase Ophthalmology, Dept. Organi di Senso, University La Sapienza,Viale del Policlinico, I55 - 0016I Rome, Italy $\mathrm{Tel}+39064997 \mid$

Fax +3906 49975304

Email alessandro.lambiase@uniromal.it
Abstract: Neurotrophic keratitis (NK) is a degenerative disease characterized by corneal sensitivity reduction, spontaneous epithelium breakdown, and impairment of corneal healing. Several causes of NK, including herpetic keratitis, diabetes, and ophthalmic and neurosurgical procedures, share the common mechanism of trigeminal damage. Diagnosis of NK requires accurate investigation of clinical ocular and systemic history, complete eye examination, and assessment of corneal sensitivity. All diagnostic procedures to achieve correct diagnosis and classification of NK, including additional examinations such as in vivo confocal microscopy, are reviewed. NK can be classified according to severity of corneal damage, ie, epithelial alterations (stage 1), persistent epithelial defect (stage 2), and corneal ulcer (stage 3). Management of NK should be based on clinical severity, and aimed at promoting corneal healing and preventing progression of the disease to stromal melting and perforation. Concomitant ocular diseases, such as exposure keratitis, dry eye, and limbal stem cell deficiency, negatively influence the outcome of NK and should be treated. Currently, no specific medical treatment exists, and surgical approaches, such as amniotic membrane transplantation and conjunctival flap, are effective in preserving eye integrity, without ameliorating corneal sensitivity or visual function. This review describes experimental and clinical reports showing several novel and potential therapies for NK, including growth factors and metalloprotease inhibitors, as well as three ongoing Phase II clinical trials.

Keywords: neurotrophic keratitis, cornea sensitivity, cornea innervation, persistent epithelial defect

\section{Definition}

Neurotrophic keratitis (NK) is a rare degenerative corneal disease caused by impairment of trigeminal innervation leading to corneal epithelial breakdown, impairment of healing, and development of corneal ulceration, melting, and perforation. ${ }^{1}$ The hallmark of NK is a decrease or absence of corneal sensation. ${ }^{1,2}$

NK was initially described as "neuroparalytic keratitis" and experimentally demonstrated by Magendie, who hypothesized the presence of trophic nerve fibers in the trigeminal nerve regulating tissue metabolism. ${ }^{3}$ It is now demonstrated that the trigeminal nerve provides corneal sensation and also supplies trophic factors to the cornea, playing a key role in maintaining the anatomical integrity and function of the ocular surface. ${ }^{4}$ The ocular surface epithelium, tear gland, and sensory and autonomic nerve fibers exert a mutual influence of their structures and functions by the release of cytokines, neuropeptides, and neuromediators. ${ }^{1,4}$ Impairment of corneal trigeminal innervation causes morphological and metabolic epithelial disturbances and leads to development of recurrent or persistent epithelial defects. 


\section{Causes}

Ocular and systemic conditions associated with damage at any level of the fifth cranial nerve, from the trigeminal nucleus to the corneal nerve endings, may cause the development of NK. The most common causes of impairment of corneal sensation are herpetic keratitis, intracranial space-occupying lesions, and/or neurosurgical procedures that damage the trigeminal ophthalmic branch. Other ocular causes of impairment of corneal sensitivity include chemical burns, physical injuries, corneal dystrophy, chronic use of topical medications, and anterior segment surgery involving nerve transection. Many systemic conditions are also associated with the development of corneal anesthesia, including diabetes, multiple sclerosis, congenital syndromes, and leprosy. ${ }^{1}$

\section{Epidemiology}

NK is classified as an orphan disease (ORPHA137596) with an estimated prevalence of less than 5/10,000 individuals. Since data on the epidemiology of NK are not available from the literature, the prevalence and incidence of NK may be estimated as being below 1.6/10,000 from the epidemiological data on conditions associated with NK, such as herpetic keratitis $(1.22 / 10,000)$ and post-surgical procedures $(0.02 / 10,000)$. In fact, NK develops in an average of $6 \%$ of herpetic keratitis cases, which have a prevalence of $149 / 100,000,{ }^{5}$ and in $12.8 \%$ of herpes zoster keratitis cases, which have a prevalence of $26 / 100,000 .{ }^{6}$ In addition, $2.8 \%$ of patients who underwent surgical procedures for trigeminal neuralgia, developed NK. Given that the prevalence of trigeminal neuralgia is $1.5 / 10,000$, the prevalence of NK for trigeminal neuralgia procedures can be estimated as $0.02 / 10,000 .{ }^{7}$ The percentage of NK cases caused by other conditions, such as diabetes, multiple sclerosis, acoustic neuroma, and congenital diseases, cannot be estimated because no data are available in the literature.

\section{Clinical presentation}

NK is characterized by corneal epithelial changes ranging from superficial punctate keratopathy to recurrent and/or persistent epithelial defects (PED) and ulcers, which may progress to stromal melting and corneal perforation. Damage to the trigeminal sensory fibers also affects tear film production due to decreased stimulation of the tear gland reflex. ${ }^{1}$ Patients with NK rarely complain of symptoms, probably due to their lack of corneal sensation. An NK classification based on severity was proposed by Mackie, who distinguished three stages $^{8}$ (Table 1 and Figure 1).

Stage $1 \mathrm{NK}$ is characterized by corneal epithelial changes with dry and cloudy corneal epithelium, presence of superficial punctate keratopathy, and corneal edema. Long-lasting NK may also show epithelial hyperplasia and irregularity, superficial neovascularization, and stromal scarring. Conjunctival epithelium damage may be observed by rose bengal staining on the inferior bulbar conjunctiva. A decrease in the breakup time was also reported.

Stage 2 NK is characterized by recurrent and/or PED with an oval or circular shape, most frequently localized at the superior half of the cornea. Usually, a PED is surrounded by an area of poorly adherent opaque and edematous epithelium that can spontaneously detach and produce an enlargement

Table I Clinical grading of neurotrophic keratitis and management

\begin{tabular}{|c|c|c|}
\hline Stage & Clinical findings & Treatments \\
\hline \multirow[t]{9}{*}{ I } & Corneal epithelial hyperplasia and irregularity & Discontinuation of all topical medications \\
\hline & Scattered small facets of dried epithelium (Gaule spots) & Use of preservative-free artificial tears \\
\hline & Superficial punctate keratopathy & Treatment of ocular surface-associated diseases \\
\hline & Rose bengal staining of the inferior conjunctiva & \\
\hline & Increased viscosity of tear mucus & \\
\hline & Decreased break-up time & \\
\hline & Superficial neovascularization & \\
\hline & Stromal scarring & \\
\hline & Dellen & \\
\hline \multirow[t]{5}{*}{ II } & Persistent corneal epithelial defect with smooth and & Corneal or scleral therapeutic contact lenses \\
\hline & rolled edges & Surgical tarsorrhaphy \\
\hline & Descemet's membrane folds and stromal swelling & Palpebral spring, botulinum A toxin injection of \\
\hline & Anterior chamber inflammatory reaction with & the eyelid elevator \\
\hline & hypopyon (rare) & Amniotic membrane transplantation \\
\hline \multirow[t]{4}{*}{ III } & Corneal ulcer & Cyanoacrylate glue followed by a soft bandage \\
\hline & Corneal perforation & contact lens \\
\hline & Corneal stromal melting & Tarsorrhaphy and conjunctival flap \\
\hline & & Lamellar or penetrating keratoplasty \\
\hline
\end{tabular}




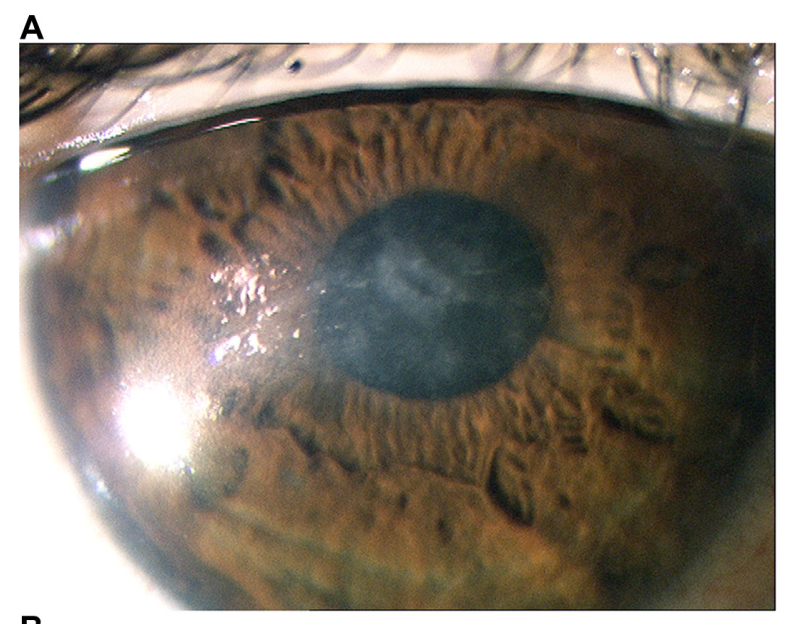

B

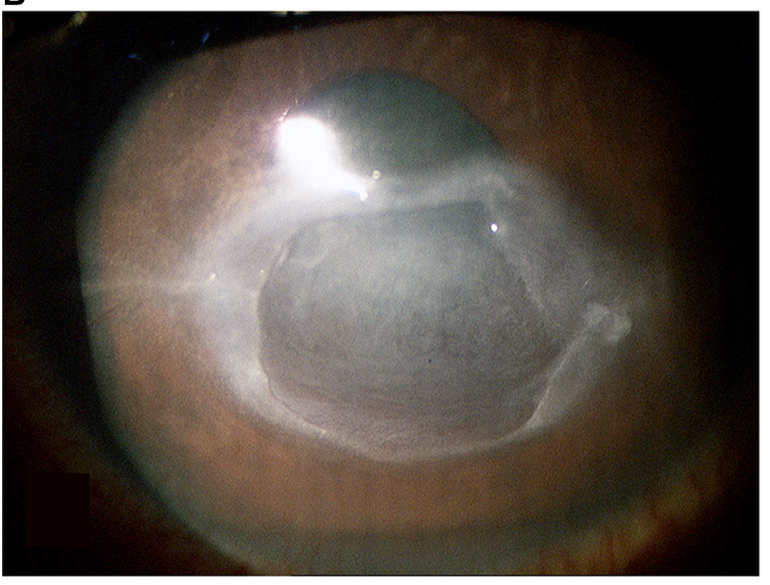

C

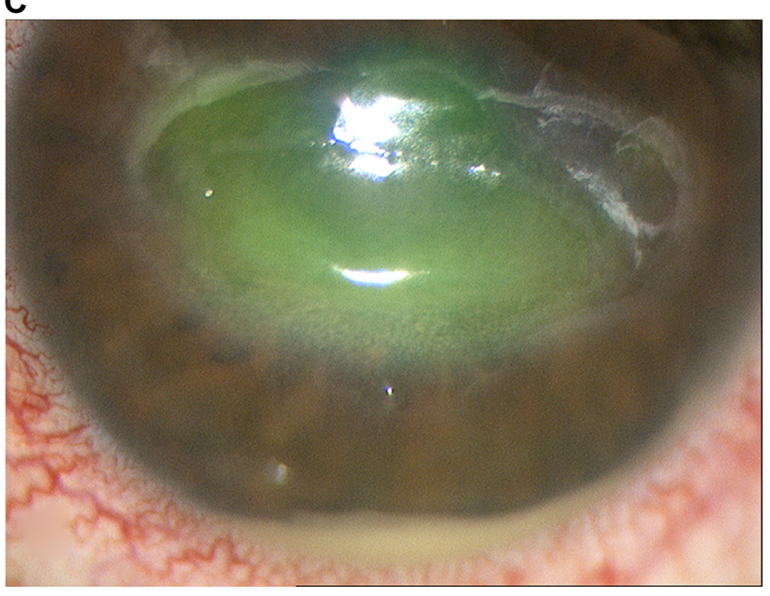

Figure I Stage I neurotrophic keratitis (A) showing cloudy and irregular corneal epithelium associated with mild stromal scarring. Stage 2 neurotrophic keratitis (B) with a large persistent epithelial defect characterized by smooth, rolled edges. No signs of ocular inflammation are present. Stage 3 neurotrophic keratitis $(\mathbf{C})$ characterized by deep corneal ulcer, stromal melting, and sterile hypopyon.

of the PED. Due to impaired epithelial healing, the edges of the PED become smooth and rolled. Descemet's membrane folds and stromal edema may be observed.

Stage 3 NK is characterized by corneal ulcer with stromal involvement that may be complicated by stromal melting and progression to corneal perforation. ${ }^{1,8}$ Rarely, an inflammatory reaction in the anterior chamber may be present and associated with sterile hypopyon.

\section{Diagnosis}

The diagnosis of NK is mainly based on the clinical history of conditions associated with trigeminal impairment, presence of PED or ulcers, and decreased corneal sensitivity. Patients with NK rarely complain of ocular symptoms, with a discrepancy between clinical findings and symptoms. Even if reduction or absence of corneal sensitivity is pathognomonic of NK, a differential diagnosis with infective and immune keratitis should always be included. On the other hand, the assessment of corneal sensitivity during evaluation for corneal ulcer may reveal the presence of NK, allowing correct diagnosis and treatment (Figure 2).

\section{Clinical history}

Many ocular and systemic conditions may be associated with development of NK and the patient's clinical history should be accurately investigated. Presence of systemic disease (diabetes or multiple sclerosis), treatments (neuroleptic and antipsychotic drugs), and local causes (previous corneal surgery or traumas, topical anesthetic abuse, chronic topical drug use, chemical burns, or contact lens abuse) potentially leading to corneal sensory impairment should be carefully investigated. Additionally, conditions that may damage the fifth cranial nerve or its nucleus, such as neoplasms of the brain, neurosurgery, or vascular accident, should be considered. Finally, NK has been described in several congenital diseases, including familial dysautonomia, Goldenhar-Gorlin syndrome, Moebius syndrome, familial corneal hypoesthesia, and congenital insensitivity to pain with anhidrosis. ${ }^{1}$

\section{Ocular symptoms}

Symptoms of ocular surface discomfort are rarely reported by NK patients. However, patients may complain of blurred vision due to PED, corneal stroma scarring, and/or swelling. ${ }^{1,8}$

\section{General examination}

General examination assessing for the presence of cranial nerve involvement may aid localization of trigeminal nerve damage. Seventh or eighth cranial nerve palsy may indicate trigeminal involvement from acoustic neuroma or from its surgical resection. Third, fourth, and sixth cranial nerve dysfunction may indicate an aneurysm or cavernous sinus that also damages the trigeminal nerve. ${ }^{1}$ 


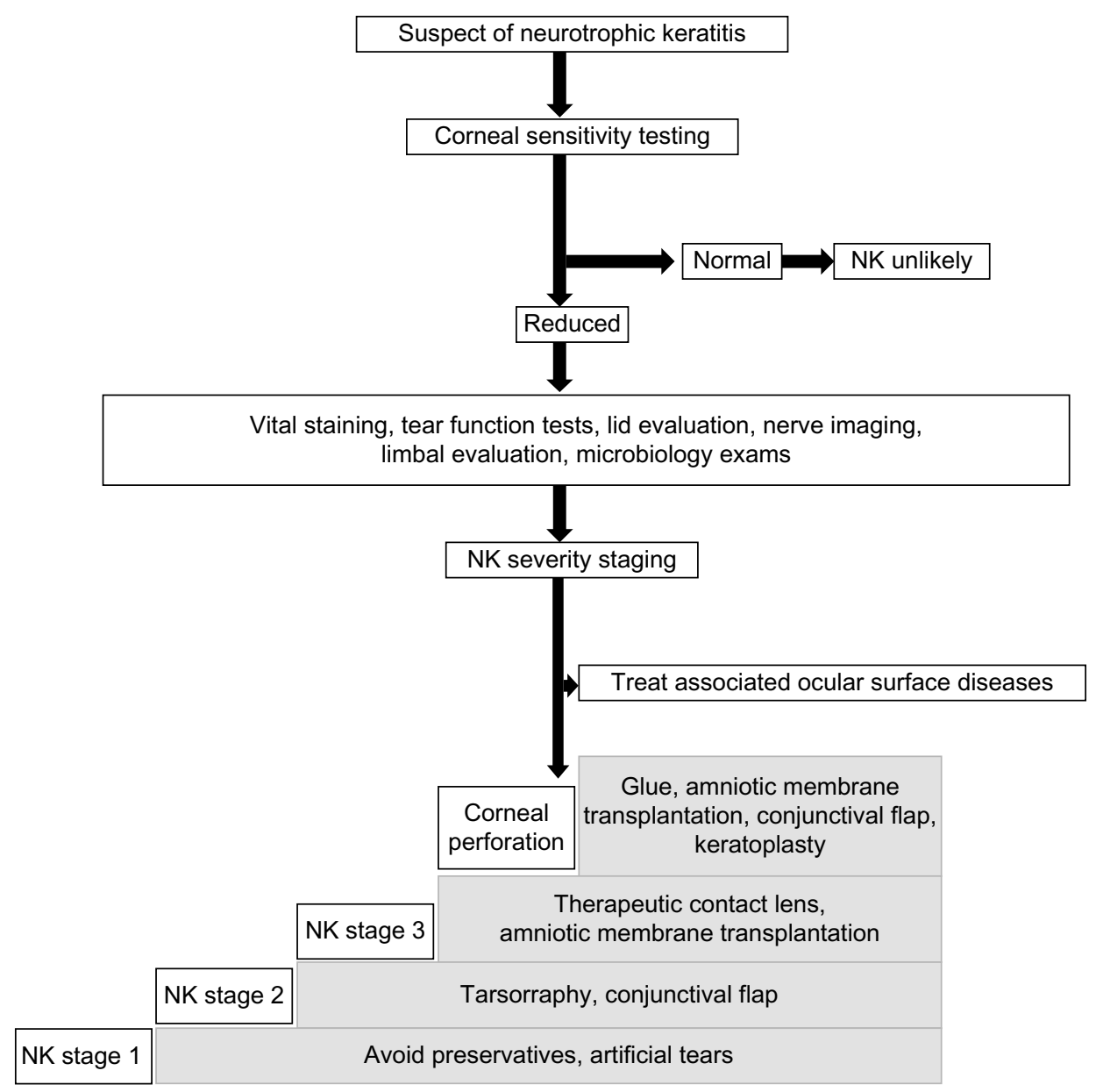

Figure 2 Stepwise approach to the diagnosis and treatment of neurotrophic keratitis (NK).

\section{Ocular examination}

The eyelids should be examined for both diagnostic and prognostic purposes; lagophthalmos may indicate an association with seventh cranial nerve palsy and may lead to exposure keratitis, worsening the clinical progression of NK. Ptosis may indicate the concomitant involvement of the third cranial nerve. Blink rate is also decreased in bilateral NK. The conjunctiva in NK patients rarely shows signs of inflammation despite the presence of corneal epithelial defects or ulcer. The presence of subconjunctival fibrosis should be noted as a sign of associated chronic autoimmune disease and/or severe dry eye. Presence of a marked inflammatory reaction and secretion may indicate an superimposed-infection that should be excluded by microbiological tests.

Corneal epithelial changes should be localized and quantified in order to classify NK severity and progression. Most often, corneal changes are present in the central area, and progression from superficial punctate keratopathy to PED and ulcer requires prompt treatment. Corneal melting should also be noted because its presence indicates worsening and progression of the condition, which may lead to corneal perforation. Other corneal changes, such as neovascularization and scarring, may indicate previous infections or recurrent corneal ulcers.

The presence of iris sector atrophy may be a sign of a previous herpetic infection. The anterior chamber should be evaluated for the presence of an inflammatory reaction and/or hypopyon, which may indicate a sterile neuroinflammatory reaction or a concomitant infection. Ocular fundus examination may reveal the presence of diabetic retinopathy, or optic nerve pallor or swelling due to an intracranial neoplasm. ${ }^{1}$

\section{Additional ocular examinations}

Several instrumental and laboratory tests may aid the diagnosis and management of NK. Some of them are mandatory to reach a prompt diagnosis (level 1), while others help identify concomitant morbidity, aid in differential diagnosis, classification of NK severity, and monitoring of disease progression and response to treatment (level 2). 


\section{Level I examinations}

Assessment of corneal sensitivity is essential to confirm the diagnosis of $\mathrm{NK}$ and to assess the severity of corneal nerve impairment. Corneal sensitivity can be measured qualitatively by touching the central and peripheral cornea with a cotton thread or quantitatively using a corneal aesthesiometer. ${ }^{9}$ When the cotton thread gently touches the cornea, normal subjects show a blink reaction and can describe the sensation of touch, while patients with loss of corneal sensitivity do not react. Different devices are available to measure corneal sensitivity. One of the most commonly used is the Cochet-Bonnet esthesiometer, which quantifies corneal sensitivity by a nylon filament of different lengths touching the cornea to elicit a blink or a patient response. Specifically, the nylon filament may be extended from 0 to $6 \mathrm{~cm}$ and each quadrant of the cornea may be tested separately. The measure of corneal sensitivity can be expressed in centimeters corresponding to the length of the nylon filament that elicits corneal sensation and blinking. ${ }^{10}$ Recently, new no-contact esthesiometers have been developed, allowing separate assessment of corneal mechanical, chemical, and thermal sensitivity. The Belmonte non-contact esthesiometer is based on corneal stimulation by air puffs at different temperatures, pressures, and concentrations of $\mathrm{CO}_{2}{ }^{11}$ In contrast with the Cochet-Bonnet esthesiometer, such non-contact instruments enable evaluation of all three types of neuroreceptors on the ocular surface.

Administration of hypertonic (3\%) saline solution by eye drops was proposed by Mandahl to evaluate ocular surface sensitivity, and showed better sensitivity than cotton wool thread, but was not better than the Cochet-Bonnet esthesiometer. ${ }^{12}$ Several vital stains are routinely used to evaluate ocular surface integrity. The most widely used is fluorescein, which allows identification of corneal damage. Lissamine green and rose bengal also allow evaluation of conjunctival epithelial integrity and to identify mucous filaments.

\section{Level 2 examinations}

Tear film function should be evaluated because decreased corneal sensitivity may alter the tear film and trigger a vicious circle in which tear film dysfunction worsens the prognosis of NK. The Schirmer test, break-up time, and tear osmolarity test may easily detect tear film changes and allow differential diagnosis with primary dry eye syndrome. ${ }^{13}$

Lack of corneal epithelial integrity is the main risk factor for development of cornea sovrainfection. Therefore, active bacterial, viral, parasitic, or fungal infection should be investigated for using microbiological examination in cases with PED or ulcers. ${ }^{1}$

In vivo confocal microscopy has recently been introduced in routine clinical evaluation to study the cornea structures at the cellular level, including the stromal and sub-basal nerves, and to provide images comparable with ex vivo histochemical methods. Different types of in vivo confocal microscopy have been used to evaluate corneal nerve morphology and changes in NK patients. ${ }^{4,14}$ A Confoscan 4 (Nidek Technologies, Fremont, CA, USA) study showed a significant decrease in the total number of sub-basal nerve fibers, and a significant correlation with decreased corneal sensation in patients with herpes zoster keratitis, herpes simplex keratitis, and post refractive surgery. ${ }^{15-17}$ In diabetic patients, a tandem scanning confocal microscope (Model 165A; Tandem Scanning, Reston, VA, USA) analysis showed a decrease in corneal sub-basal nerve fiber density, increased nerve tortuosity, and a significant correlation with the severity of peripheral neuropathy. ${ }^{18,19}$ Patients with NK caused by monolateral neurosurgical trigeminal damage showed decreased corneal sensitivity which correlated with the decreased number and density of corneal nerve fibers. Interestingly, this study showed that morphological changes occur in all corneal structures. Specifically, we observed a decrease in corneal epithelial and endothelial cell density and an increase in the number of hyper-reflective keratocytes in NK eyes when compared with healthy contralateral eyes. In addition, the lower endothelial cell density significantly correlated with a longer duration of NK, suggesting that the trigeminal nerve also plays a role in supporting survival and function of the corneal endothelium. ${ }^{20}$ The Heidelberg Retina Tomograph 2 Rostock Cornea Module laser scanning confocal microscope (Heidelberg Engineering, Carlsbad, CA, USA) showed improvement of corneal innervation after treatment with topical autologous plasma in patients with NK. ${ }^{21}$

The major limitation of corneal nerve evaluation is its poor repeatability, which is related to the manual qualitative analysis of nerve parameters. Algorithms for automatic tracing and evaluation of the corneal nerve are in development. ${ }^{22,23}$ Testing of corneal sensitivity and imaging of the corneal nerves may be useful to aid the diagnosis of NK and may also assist in management of the condition.

Ocular surface impression cytology of the cornea and conjunctiva may be a useful additional examination to characterize the conjunctival epithelial changes related to dry eye and/or corneal conjunctivalization as observed in limbal stem cell deficiency. ${ }^{24}$ Both dry eye and limbal stem cell 
deficiency may be secondary to NK, but can also be comorbidities that negatively influence the clinical outcome.

Optimal management of NK frequently requires consultation with other specialists (eg, neurologist, otorhinolaryngologist, diabetologist) and cranial imaging, blood examinations, and electrofunctional evaluations. A simple check of facial sensitivity may indicate concomitant involvement of the second and third branch of the trigeminal nerve.

\section{Differential diagnosis}

The diagnosis of NK is easily oriented by the history and pathognomonic evidence of corneal lesions associated with poor symptomatology and impairment of corneal sensitivity. However, several chronic diseases, such as dry eye syndrome, blepharitis, exposure keratopathy, topical drug toxicity, mild chemical injury, contact lens-related disorders, and corneal limbal stem cell deficiency, may impair corneal sensitivity or be comorbidities in patients with NK. Level 2 examinations aid identification of coexisting pathological conditions, such as tear film alterations or limbal stem cell deficiency, that should be treated to avoid progression of NK.,25

The presence of a corneal ulcer requires exclusion of other common causes, such as infectious or immune disease. Localization and the characteristics of the corneal lesion, as well as the presence of stromal infiltrates associated with important ocular inflammation, make it mandatory to perform microbiological and immunological evaluations. Any local treatment should be discontinued to exclude drug-induced corneal ulcers. ${ }^{1,26}$

\section{Management}

Early diagnosis, severity-based treatment, and careful monitoring of NK patients are mandatory to achieve epithelial healing and prevent progression of corneal damage, especially since worsening of $\mathrm{NK}$ is frequently asymptomatic (Table 1).

All topical medications should be discontinued because they have detrimental effects on the ocular surface epithelium. Additionally, all ocular surface-associated diseases, such as exposure keratitis, dry eye, and limbal stem cell deficiency, which may worsen the prognosis of NK, need to be taken care of. For instance, it will be necessary to correct any eyelid dysfunction, to consider punctal occlusion, and/or perform limbal cell transplantation. ${ }^{1,25}$

Treatment of NK should be based on disease severity. Treatment for stage 1 disease aims at improving epithelial quality and transparency and avoiding epithelial breakdown. In the presence of PED, therapy is aimed at preventing stromal involvement and corneal ulcer formation as well as promoting corneal healing. More severe cases, with corneal ulcer and stromal melting, require immediate attention to stop the stromal lysis and prevent perforation ${ }^{1,25,27}$ (Table 1).

Pharmacological treatments for NK are not available. However, the use of preservative-free artificial tears may help improve the corneal surface at all stages of disease severity. Topical steroids have been proposed for NK to control ocular inflammation (if present); however, steroids may increase the risk of corneal melting and perforation by inhibiting stromal healing, and their use should be considered with great caution. ${ }^{1,25,28}$ Topical nonsteroidal antiinflammatory drugs may also inhibit the healing process and should be avoided. ${ }^{29}$ In the event of stromal melting, use of topical collagenase inhibitors, such as $\mathrm{N}$-acetylcysteine, and systemic administration of tetracycline or medroxyprogesterone may be considered. ${ }^{30-32}$ Use of topical antibiotic eye drops to prevent infection in eyes with NK at stages 2 and 3 is recommended.

Nonpharmacological treatments for NK include therapeutic corneal or scleral contact lenses in the event of PED to promote corneal epithelial healing. ${ }^{33}$ Contact lens use may increase the risk of secondary infections.

Surgical treatments are reserved for refractory cases. Partial or total tarsorrhaphy is the most simple and widespread procedure used to promote corneal healing in the presence of a PED. Surgical tarsorrhaphy may be performed easily and the tarsorrhaphy opening may be enlarged a few weeks after corneal healing. Opening the tarsorrhaphy prematurely may result in recurrence of PED. Alternatively, it is possible to use a palpebral spring or botulinum A toxin injection of the eyelid elevator muscle to cover the PED and promote healing. ${ }^{34,35}$

Amniotic membrane transplantation (AMT) is a surgical technique for ocular surface reconstruction that should be considered in the management of refractory neurotrophic corneal ulcers. AMT is relatively easy to perform, and is effective in promoting corneal epithelial healing, reducing vascularization, and reducing ocular surface inflammation. Multilayer AMT has been proposed for treating deep neurotrophic corneal ulcers. ${ }^{34-36} \mathrm{~A}$ randomized controlled trial comparing AMT with conventional treatments in eyes with refractory NK showed that both AMT and conventional management (tarsorrhaphy versus bandage contact lens) were effective treatments for NK, with a median time to complete epithelialization of 21 days in both groups. ${ }^{35,36}$ TachoSil (Nycomed Pharma, Zurich, Switzerland), an absorbable 
fibrin sealant patch, with or without AMT, has also been used successfully to treat noninfective corneal perforation. ${ }^{37,38}$

The conjunctival flap is a standard surgical procedure able to restore ocular surface integrity and provide metabolic and mechanical support for corneal healing. This procedure is effective for the treatment of chronic corneal ulceration with or without corneal perforation..$^{39,40}$ The ulcer/perforation is covered by a pedunculated conjunctival flap secured in place by fine sutures. ${ }^{41}$ The main goal of this procedure is to preserve the anatomical integrity of the eye, but it does not restore visual function.

Both tarsorrhaphy and the conjunctival flap are effective surgical procedures for promoting corneal healing, but have a poor cosmetic outcome and visual function is sacrificed. In cases with corneal perforation, small perforations $(<3 \mathrm{~mm})$ can be treated by application of cyanoacrylate glue to the site of perforation, followed by application of a soft bandage contact lens or AMT. ${ }^{42}$ Larger defects require a conjunctival flap or lamellar/penetrating keratoplasty. The success rate of corneal transplants in NK patients is low due to the lack of trophic support, with consequent poor wound healing and risk of PED recurrence. ${ }^{43,44}$

\section{Prognosis}

NK is one of the most difficult and challenging ocular diseases that lack a specific treatment. The prognosis of NK depends on the cause and severity of the trigeminal damage and on the presence of associated ocular surface disease (such as dry eye or exposure keratitis). It is generally accepted that the more severe the corneal sensory impairment, the higher the probability of NK progression. Treatment of NK should be conservative. Corneal transplantation aimed at restoring corneal transparency should be discouraged because of the high risk of PED, ulcers, corneal melting, and perforation after surgery.

\section{Future developments}

Novel medical and surgical treatments have been proposed for NK and are currently under investigation. ${ }^{1,25,45}$ Surgical procedures of sensory neurotization by insertion of the contralateral supraorbital and supratrochlear nerves at the anesthetic corneal limbus have been reported to improve the outcome in six NK patients with unilateral facial palsy and corneal anesthesia. ${ }^{46}$ The use of a Boston keratoprosthesis (Boston K-Pro; Massachusetts Eye and Ear Infirmary, Boston, MA, USA) restored visual function at 4-month follow-up in one patient with NK caused by herpes zoster keratitis. ${ }^{47}$

Open studies have reported the efficacy of several pharmacological treatments in promoting corneal ulcer healing in NK. Treatment with eye drops containing RGTA ${ }^{\circledR}$
(Cacicol20 ${ }^{\circledR}$; OTR3, Paris, France), a matrix agent mimicking heparan sulfate, achieved complete healing in eight of eleven patients $(73 \%)$, with closing of the NK ulcer after a mean period of 8.7 weeks. $^{48}$

In another study, nine NK patients with PED were treated with eye drops containing thymosin beta-4 (a synthetically produced copy of the naturally occurring, 43-amino acid, $\mathrm{G}$-actin sequestering molecule). One of these patients had completely healed by the end of the treatment period, and three patients had healed by the end of 30 days of follow-up. ${ }^{49}$

Autologous serum (50\%) eye drops induced corneal healing in 23 of 25 eyes with $\mathrm{NK}$ in a mean time of 22 days. ${ }^{50}$ Further, the efficacy of autologous serum eye drops and AMT was compared in a retrospective study that showed both treatments to be effective and that multilayer AMT was more effective for treating deep corneal ulcers after herpes simplex keratitis. ${ }^{51}$

Treatment of patients with PED using topical non-gelified platelet-rich plasma caused by several conditions, including NK, achieved complete healing in all treated eyes within 6-32 days. $^{52}$ In addition, 28 patients with NK showed complete corneal healing after a mean 4 weeks' treatment using umbilical cord serum eye drops. ${ }^{53}$

Several studies have proposed the use of neuropeptides and growth factors for the treatment of NK. However, preliminary data on the successful use of epidermal growth factor eye drops in patients with NK were not confirmed in larger studies. ${ }^{54}$

Topical treatment with substance $\mathrm{P}$ and insulin-like growth factor-1 was used in 25 patients with NK in an open study, with complete healing of epithelial defects observed in $73 \%$ of patients within 4 weeks. ${ }^{55}$ Subsequently, a combination of substance P-derived peptide, phenylalanine-glycine-leucine-methionine-amide, and insulin-like growth factor-1 was used to treat PED in eleven patients with NK, and achieved complete epithelial resurfacing in $89 \%$ of cases. ${ }^{56}$ Nerve growth factor eye drops represent a most promising future therapeutic approach for the prevention and treatment of NK. Nerve growth factor is able to improve tear and nerve function and to stimulate regeneration and survival of both the corneal epithelium and sensory nerves, potentially leading to recovery from NK. Treatment with topical nerve growth factor was also effective in patients with moderate to severe NK (stages 2 and 3). Two open uncontrolled studies reported complete corneal healing in $100 \%$ of 55 patients after 12 days to 6 weeks of treatment with nerve growth factor eye drops. 
Corneal scarring was also decreased and improvement of visual acuity was observed. Restoration of corneal integrity was associated with recovery of corneal sensitivity in most patients. ${ }^{57-59}$

Recently, a recombinant human nerve growth factor eye drop formulation has been developed for clinical use, and Phase I studies have shown it to be safe and well tolerated. ${ }^{60}$ Three Phase II clinical trials are ongoing to evaluate the safety and efficacy of novel therapies for NK patients, including recombinant human nerve growth factor (ClinicalTrials. gov identifiers NCT01756456, NCT01794312, and NCT01165450). ${ }^{61-63}$

\section{Conclusion}

The diagnosis and management of NK is a challenge for ophthalmologists. The currently available medical and surgical treatments aim to promote healing, prevent disease progression, and avoid corneal perforation. However, no therapies are currently available to improve the impairment of corneal sensitivity and restore visual acuity. Experimental and clinical evidence shows that several novel treatments, such as growth factors, may improve the clinical outcome of NK. The findings of presently ongoing clinical trials will enable new therapies to be introduced for this orphan disease.

\section{Disclosure}

The authors report no conflicts of interest in this work.

\section{References}

1. Bonini S, Rama P, Olzi D, Lambiase A. Neurotrophic keratitis. Eye (Lond). 2003;17:989-995.

2. Groos EB Jr. Neurotrophic keratitis. In: Krachmer JH, Mannis MJ, Holland EJ, editors. Cornea: Clinical Diagnosis and Management. St Louis, MO, USA: Mosby; 1997.

3. Magendie F. De l'influence de la cinquieme paire de nerfs sur la nutrition et les fonctions de l'oeil. [The influence of the fifth pair of nerves on nutrition and function of the eye]. J Physiol Exp Pathol. 1824;4:176-182. French.

4. Müller LJ, Marfurt CF, Kruse F, Tervo TM. Corneal nerves: structure, contents and function. Exp Eye Res. 2003;76:521-542.

5. Labetoulle M, Auquier P, Conrad H, et al. Incidence of herpes simplex virus keratitis in France. Ophthalmology. 2005;112:888-895.

6. Dworkin RH, Johnson RW, Breuer J, et al. Recommendations for the management of herpes zoster. Clin Infect Dis. 2007;44 Suppl 1: S1-S26.

7. Bhatti MT, Patel R. Neuro-ophthalmic considerations in trigeminal neuralgia and its surgical treatment. Curr Opin Ophthalmol. 2005;16: 334-340.

8. Mackie IA. Neuroparalytic keratitis. In: Fraunfelder F, Roy FH, Meyer SM, editors. Current Ocular Therapy. Philadelphia, PA, USA: WB Saunders; 1995.

9. Faulkner WJ, Varley GA. Corneal diagnostic technique. In: Krachmer JH, Mannis MJ, Holland EJ, editors. Cornea: Fundamentals of Corneal and External Disease. St Louis, MO, USA: Mosby; 1997.
10. Norn MS. Measurement of sensitivity. In: Norn MS, editor. External Eye Diseases. Methods of Examination. Copenhagen, Denmark: Munksgaard International Publisher Ltd; 1974.

11. Belmonte C, Acosta MC, Schmelz M, Gallar J. Measurement of corneal sensitivity to mechanical and chemical stimulation with a $\mathrm{CO}_{2}$ esthesiometer. Invest Ophthalmol Vis Sci. 1999;40:513-519.

12. Mandahl A. Hypertonic saline test for ophthalmic nerve impairment. Acta Ophthalmol (Copenh). 1993;71:556-559.

13. Heigle TJ, Pflugfelder SC. Aqueous tear production in patients with neurotrophic keratitis. Cornea. 1996;15:135-138.

14. Patel DV, McGhee CN. In vivo confocal microscopy of human corneal nerves in health, in ocular and systemic disease, and following corneal surgery: a review. Br J Ophthalmol. 2009;93:853-860.

15. Hamrah P, Cruzat A, Dastjerdi MH, et al. Unilateral herpes zoster ophthalmicus results in bilateral corneal nerve alteration: an in vivo confocal microscopy study. Ophthalmology. 2013;120:40-47.

16. Hamrah P, Cruzat A, Dastjerdi MH, et al. Corneal sensation and subbasal nerve alterations in patients with herpes simplex keratitis: an in vivo confocal microscopy study. Ophthalmology. 2010;117:1930-1936.

17. Linna TU, Vesaluoma MH, Pérez-Santonja JJ, Petroll WM, Alió JL, Tervo TM. Effect of myopic LASIK on corneal sensitivity and morphology of subbasal nerves. Invest Ophthalmol Vis Sci. 2000;41: 393-397.

18. Rosenberg ME, Tervo TM, Immonen IJ, et al. Corneal structure and sensitivity in type 1 diabetes mellitus. Invest Ophthalmol Vis Sci. 2000;41:2915-2921.

19. Cruzat A, Pavan-Langston D, Hamrah P. In vivo confocal microscopy of corneal nerves: analysis and clinical correlation. Semin Ophthalmol. 2010;25:171-177.

20. Lambiase A, Sacchetti M, Mastropasqua A, Bonini S. Corneal changes in neurosurgically induced neurotrophic keratitis. JAMA Ophthalmol. 2013;131:1547-1553.

21. Scarpa F, Zheng X, Ohashi Y, Ruggeri A. Automatic evaluation of corneal nerve tortuosity in images from in vivo confocal microscopy. Invest Ophthalmol Vis Sci. 2011;16;52:6404-6408.

22. Rao K, Leveque C, Pflugfelder SC. Corneal nerve regeneration in neurotrophic keratopathy following autologous plasma therapy. $\mathrm{Br} J$ Ophthalmol. 2010;94:584-591.

23. Parissi M, Karanis G, Randjelovic S, et al. Standardized baseline human corneal subbasal nerve density for clinical investigations with laser-scanning in vivo confocal microscopy. Invest Ophthalmol Vis Sci. 2013;54:7091-7102.

24. Sacchetti M, Lambiase A, Cortes M, et al. Clinical and cytological findings in limbal stem cell deficiency. Graefes Arch Clin Exp Ophthalmol. 2005;243:870-876.

25. Lambiase A, Rama P, Aloe L, Bonini S. Management of neurotrophic keratopathy. Curr Opin Ophthalmol. 1999;10:270-276.

26. Sacchetti M, Lambiase A, Coassin M, Bonini S, Bonini S. Toxic corneal ulcer: a frequent and sight-threatening disease. Eur J Ophthalmol. 2009;19:916-922.

27. Nishida T, Yanai R. Advances in treatment for neurotrophic keratopathy. Curr Opin Ophthalmol. 2009;20:276-281.

28. Petroutsos G, Guimaraes R, Giraud JP, Pouliquen Y. Corticosteroids and corneal epithelial wound healing. Br J Ophthalmol. 1982;66: 705-708.

29. Hersh PS, Rice BA, Baer JC, et al. Topical nonsteroidal agents and corneal wound healing. Arch Ophthalmol. 1990;108:577-583.

30. Hossain P. The corneal melting point. Eye (Lond). 2012;26: 1029-1030.

31. Perry HD, Golub LM. Systemic tetracyclines in the treatment of noninfected corneal ulcers: a case report and proposed new mechanism of action. Ann Ophthalmol. 1985;17:742-744.

32. Hicks CR, Crawford GJ. Melting after keratoprosthesis implantation: the effects of medroxyprogesterone. Cornea. 2003;22:497-500.

33. Grey F, Carley F, Biswas S, Tromans C. Scleral contact lens management of bilateral exposure and neurotrophic keratopathy. Cont Lens Anterior Eye. 2012;35:288-291. 
34. Portnoy SL, Insler MS, Kaufman HE. Surgical management of corneal ulceration and perforation. Surv Ophthalmol. 1989;34:47-58.

35. Khokhar S, Natung T, Sony P, et al. Amniotic membrane transplantation in refractory neurotrophic corneal ulcers: a randomized, controlled clinical trial. Cornea. 2005;24:654-660.

36. Kruse FE, Rohrschneider K, Völcker HE. Multilayer amniotic membrane transplantation for reconstruction of deep corneal ulcers. Ophthalmology. 1999;106:1504-1510.

37. Hurtado-Sarrió M, Duch-Samper A, Cisneros-Lanuza A, Díaz-Llopis M. Tachosil: a new alternative for the treatment of non-traumatic corneal perforations. Br J Ophthalmol. 2009;93:1410-1411.

38. Grau AE, Durán JA. Treatment of a large corneal perforation with a multilayer of amniotic membrane and Tachosil. Cornea. 2012;31:98-100.

39. Gundersen T, Pearlson HR. Conjunctival flaps for corneal diseases: their usefulness and complications. Trans Am Ophthalmol Soc. 1969;67:78-95.

40. Lugo M, Arentsen JJ. Treatment of neurotrophic ulcers with conjunctival flaps. Am J Ophthalmol. 1987;103:711-712.

41. Khodadoust A, Quinter AP. Microsurgical approach to the conjunctival flap. Arch Ophthalmol. 2003;121:1189-1193.

42. Golubović S, Parunović A. [Cyanoacrylate glue in the treatment of corneal ulcerations]. Fortschr Ophthalmol. 1990;87:378-381. German.

43. Hirst LW, Smiddy WE, Stark WJ. Corneal perforations: changing methods of treatment, 1960-1980. Ophthalmology. 1982;89:630-634.

44. Jhanji V, Young AL, Mehta JS, Sharma N, Agarwal T, Vajpayee RB. Management of corneal perforation. Surv Ophthalmol. 2011;56: 522-538.

45. Semeraro F, Forbice E, Romano V, et al. Neurotrophic keratitis. Ophthalmologica. October 2, 2013. [Epub ahead of print.]

46. Terzis JK, Dryer MM, Bodner BI. Corneal neurotization: a novel solution to neurotrophic keratopathy. Plast Reconstr Surg. 2009;123:112-120.

47. Pavan-Langston D, Dohlman CH. Boston keratoprosthesis treatment of herpes zoster neurotrophic keratopathy. Ophthalmology. 2008; 115(Suppl 2):S21-S23.

48. Aifa A, Gueudry J, Portmann A, Delcampe A, Muraine M. Topical treatment with a new matrix therapy agent (RGTA) for the treatment of corneal neurotrophic ulcers. Invest Ophthalmol Vis Sci. 2012;53:

49. Dunn SP, Heidemann DG, Chow CY, et al. Treatment of chronic nonhealing neurotrophic corneal epithelial defects with thymosin beta4 Ann N Y Acad Sci. 2010;1194:199-206.

50. Jeng BH, Dupps WJ Jr. Autologous serum 50\% eye drops in the treatment of persistent corneal epithelial defects. Cornea. 2009;28:1104-1108.

51. Turkoglu E, Celik E, Alagoz G. A comparison of the efficacy of autologous serum eye drops with amniotic membrane transplantation in neurotrophic keratitis. Semin Ophthalmol. June 12, 2013. [Epub ahead of print.] $8181-8185$.

52. Geremicca W, Fonte C, Vecchio S. Blood components for topical use in tissue regeneration: evaluation of corneal lesions treated with platelet lysate and considerations on repair mechanisms. Blood Transfus. 2010;8:107-112.

53. Yoon KC, You IC, Im SK, Jeong TS, Park YG, Choi J. Application of umbilical cord serum eyedrops for the treatment of neurotrophic keratitis. Ophthalmology. 2007;114:1637-1642.

54. Daniele S, Gilbard JP, Schepens CL. Treatment of persistent epithelial defects in neurotrophic keratitis with epidermal growth factor: a preliminary open study. Graefes Arch Clin Exp Ophthalmol. 1992;230: 314-317.

55. Yamada N, Matsuda R, Morishige N, et al. Open clinical study of eye-drops containing tetrapeptides derived from substance $\mathrm{P}$ and insulin-like growth factor-1 for treatment of persistent corneal epithelial defects associated with neurotrophic keratopathy. $\mathrm{Br} J$ Ophthalmol. 2008;92:896-900.

56. Nishida T, Chikama T, Morishige N, Yanai R, Yamada N, Saito J. Persistent epithelial defects due to neurotrophic keratopathy treated with a substance P-derived peptide and insulin-like growth factor 1. Jpn J Ophthalmol. 2007;51:442-447.

57. Lambiase A, Rama P, Bonini S, Caprioglio G, Aloe L. Topical treatment with nerve growth factor for corneal neurotrophic ulcers. N Engl J Med. 1998;338:1174-1180.

58. Bonini S, Lambiase A, Rama P, Caprioglio G, Aloe L. Topical treatment with nerve growth factor for neurotrophic keratitis. Ophthalmology. 2000;107:1347-1351.

59. Lambiase A, Sacchetti M, Bonini S. Nerve growth factor therapy for corneal disease. Curr Opin Ophthalmol. 2012;23:296-302.

60. Ferrari MP, Mantelli F, Sacchetti M, et al. Safety and pharmacokinetics of escalating doses of human recombinant nerve growth factor eye drops in a double-masked, randomized clinical trial. BioDrugs. December 11, 2013. [Epub ahead of print.]

61 Dompé s.p.a. Evaluation of Safety and Efficacy of rhNGF in Patients With Stage 2 and 3 Neurotrophic Keratitis. (REPARO). Available from: http://clinicaltrials.gov/show/NCT01756456. NLM identifier: NCT01756456. Accessed February 18, 2014.

62. Laboratoires Thea. Efficacy and Safety Assessment of T4020 Versus Vehicule in Patients With Chronic Neurotrophic Keratitis or Corneal Ulcer. Available from: http://clinicaltrials.gov/ct2/show/NCT01794312. NLM identifier: NCT01794312. Accessed February 18, 2014.

63. University of California, San Francisco. Efficacy and Safety Study of Nexagon for Persistent Corneal Epithelial Defects (NTX-PED-001). Available from: http://clinicaltrials.gov/show/NCT01165450. NLM identifier: NCT01165450. Accessed February 18, 2014.

\section{Dovepress}

\section{Publish your work in this journal}

Clinical Ophthalmology is an international, peer-reviewed journal covering all subspecialties within ophthalmology. Key topics include: Optometry; Visual science; Pharmacology and drug therapy in eye diseases; Basic Sciences; Primary and Secondary eye care; Patient Safety and Quality of Care Improvements. This journal is indexed on Submit your manuscript here: http://www.dovepress.com/clinical-ophthalmology-journal
PubMed Central and CAS, and is the official journal of The Society of Clinical Ophthalmology (SCO). The manuscript management system is completely online and includes a very quick and fair peer-review system, which is all easy to use. Visit http://www.dovepress.com/ testimonials.php to read real quotes from published authors. 